\title{
Biomimetic MEMS Directional Microphone Structures for Multi-Band Operation
}

\author{
Yansheng Zhang, James F.C. Windmill, Deepak Uttamchandani \\ University of Strathclyde \\ Glasgow, United Kingdom \\ Email: yansheng.zhang.101@strath.ac.uk
}

\begin{abstract}
Researchers have developed a variety of microelectromechinical systems (MEMS) directional microphones inspired by the behavior of the hearing organ of the parasitoid fly Ormia ochracea. Ormia's hearing organ can detect a sound's direction as accurately as the human auditory system despite its small overall size of less than $1 \mathrm{~mm}$. This remarkable capability has led to in-depth studies of its mechanics which, in turn, have led to the practical realization of several MEMS based directional acoustic sensors. The majority of earlier designs were limited to one frequency band and have relatively complicated structures for manufacture. A new MEMS microphone design described in this paper expands the simultaneous working frequency bands and reduces the cost of device fabrication without significantly increasing overall dimensions.
\end{abstract}

Keywords-Ormia ochracea, directional microphone, multibands, biological inspired, MEMS

\section{INTRODUCTION}

The human auditory system distinguishes the direction and origin of sound based on the inter-aural time difference of arrival (TDOA) and intensity difference (ID). Similar to the human auditory system, existing designs of directional microphone systems are implemented either by using an omnidirectional microphone array arranged in specific intervals to acquire directional cues (i.e. TDOA between two adjacent microphones), or a diaphragm where two surfaces are exposed to the sound propagation medium, thereby allowing the system to detect TDOA and ID depending on the difference in sound travel paths to the two surfaces. Directional microphones have been widely designed for micro-scale applications, such as hearing aids and mobile gadgets. This means that the microphones should be small enough to be embedded in such applications. However, the standard technique of relying on TDOA and ID has dimensional limitations for micro-scale applications. Assuming that the directional microphone is not affected by diffraction and phase interference, then at frequencies below $10 \mathrm{kHz}$ where most human voice energy is confined the sensor part of the system may not be larger than 4 $\mathrm{mm}$ in radius [1]. Such a small size results in almost zero TDOA and ID when the system responds to human voice frequencies. In contrast, if the device is larger than this critical size, it is still combined with a large degree of complex post signal processing since the far-field assumption is typically no longer valid. In order to achieve a micro-scale directional microphone, and reduce the cost of post processing, groups of scientists and researchers have imitated the mechanical structure of the hearing organ of Ormia ochracea, a parasitic insect, to design biological inspired directional microphones which only require minimal signal processing and are smaller than the critical size, by using MEMS fabrication processes.

In nature, the female Ormia ochracea fly locates and oviposits on male field crickets, depending on their host's mating calls [2]. The separation between its two tympana is around $520 \mu \mathrm{m}$, providing a TDOA of approximately $1.5 \mu \mathrm{s}$ and an inter-aural ID of $1 \mathrm{~dB}$ when the two tympana are regarded as isolated receivers. With a piece of stiff cuticular tissue connecting the tympana, the tympanal arrangement forms a mechanical coupled structure that enhances the ID and TDOA to $13 \mathrm{~dB}$ and $50 \mu \mathrm{s}$, respectively. The coupled structure also adopts two main vibration modes which contribute to the motion of the diaphragm. One is a rocking mode where the two tympana move out of phase, while the other is a bending mode in which the two tympana vibrate in phase. Miles, et al. [3] designed microphones inspired by the Ormia system that had a polysilicon diaphragm with two cross stiffeners rotating along a T-section beam. This design primarily locates sound direction over a narrow frequency band around the resonance frequency of the rocking mode by establishing a relationship between the sound azimuth angle and vibration intensity of the diaphragms. Touse et al.'s design [4] is similar to Mile et al.'s design, but with asymmetric diaphragms generating two frequency bands. Another group of researchers, Miao Yu, et al. [5], have realized two clamped circular polysilicon membranes linked by a $\mathrm{SiO}_{2} / \mathrm{Si}_{3} \mathrm{~N}_{4}$ bridge. This arrangement determines azimuth angle at $8 \mathrm{kHz}$ by measuring the phase difference between the two membranes. Although the above designs locate a sound source based on different measured parameters, they are limited working in one or two specific frequency bands. Furthermore, the complex structure and multiple materials used increases the probability of defects occurring in a fabricated device. Within this overall context, a new bio-inspired microphone design model reported in this paper aims to increase the number of simultaneously working frequency bands below $10 \mathrm{kHz}$, using the simplest possible micro-mechanical structure.

\section{DESIGN AND PRINCIPLES}

One method of making Ormia-inspired directional microphones for operation at four frequency bands is to arrange four identical existing designs, e.g. Miles et al.'s design or Yu et al.'s design, with the rotation axes in the same direction. Each device then senses one frequency band. This 
method trades a larger size of overall device for more working bands. An alternative method is to use two of the microphones designed by Touse et al. However, the distance between the two devices results in an angular shift in amplitude relating to the azimuth angle on each device. The novel design presented here comprises two concentric diaphragms. Each diaphragm covers two frequency bands. The device is designed to concentrate on detecting a human voice $(<10 \mathrm{kHz})$, so the dimension of the outer square frame diaphragm was set to 2.6 $\mathrm{mm} \times 2.6 \mathrm{~mm}$ which is less than the critical $4 \mathrm{~mm}$ radius that is desirable for practical MEMS microphones. A circular diaphragm $(\mathrm{r}=1 \mathrm{~mm})$ is inserted centrally on a fixed-end beam as shown in Fig. 1, making the model rotate along the central torsion beams when responding to an incident sound wave. To reduce the complexity of fabrication, the SOIMUMPS process supplied by the MEMSCAP [6] manufacture service is utilized to produce this device. This provides a silicon-on-insulator wafer with a $\mathrm{SiO}_{2}$ layer which separates a $10 \mu \mathrm{m}$ thick n-type doped single-crystal-silicon layer (the device layer) from a $400 \mu \mathrm{m}$ thickness supporting substrate (the handle). The SOIMUMPS process offers deep reaction ion etching (DRIE) from the underside of the substrate which has a beneficial outcome, as it avoids squeeze film damping.

To ensure that the efficient working frequency bands lie in the frequency range of interest, a four-degree-of-freedom spring-mass equivalent mechanical model has been constructed to describe the system performance and infer the first four resonance frequencies (see Fig. 2). Ignoring the kinetic energy of the short length of torsional beams, the kinetic energy $\mathrm{T}$ of the whole system is given by

$T=\frac{1}{2} m_{1} \dot{x}_{1}^{2}+\frac{1}{2} m_{2} \dot{x}_{2}^{2}+\frac{1}{2} m_{3} \dot{x}_{3}^{2}+\frac{1}{2} m_{4} \dot{x}_{4}^{2}$,

where $\mathrm{m}$ and $\mathrm{x}$ are the mass and the displacement field of each wing (one side of a diaphragm), respectively. The subscripts represent the corresponding number shown in Fig. 1. The potential energy $\mathrm{V}$ in the system is equal to the sum of energy generated by the inherent stiffness $\mathrm{k}_{\mathrm{i}}$ of each wing and the coupling stiffness $\mathrm{k}_{\mathrm{tij}}$ between two adjacent parts of diaphragms:

$V=\frac{1}{2} k_{1} x_{1}^{2}+\frac{1}{2} k_{2} x_{2}^{2}+\frac{1}{2} k_{3} x_{3}^{2}+\frac{1}{2} k_{4} x_{4}^{2}+\frac{1}{2} k_{t 12}\left(x_{1}+x_{2}\right)^{2}+$

$\frac{1}{2} k_{t 34}\left(x_{3}+x_{4}\right)^{2}+\frac{1}{2} k_{t 14}\left(x_{1}+x_{4}\right)^{2}+\frac{1}{2} k_{t 23}\left(x_{2}+x_{3}\right)^{2}+$

$\frac{1}{2} k_{t 13}\left(x_{1}-x_{3}\right)^{2}+\frac{1}{2} k_{t 24}\left(x_{2}-x_{4}\right)^{2}$

Applying Lagrange's equation and deriving the eigenvalues, the equations of the four natural frequencies can be written as follows:

$f_{1}, f_{2}=\frac{1}{2 \pi} \sqrt{\frac{\left(C+K_{i}\right) M_{i}+\left(C+K_{o}\right) M_{o} \mp \sqrt{(A C+E)^{2}+4 B C^{2}}}{2 B}}$,

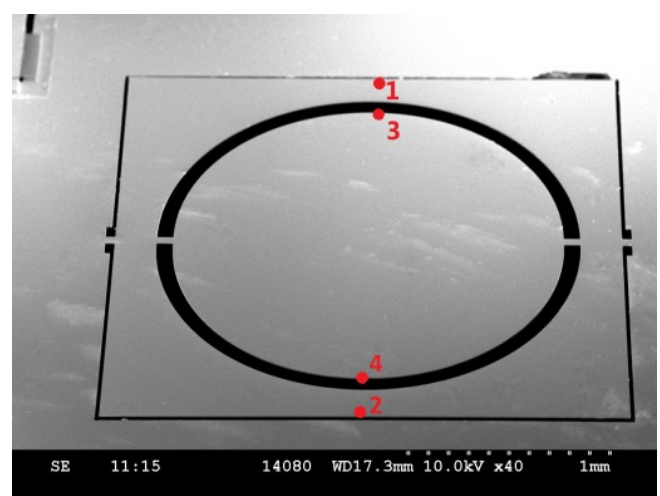

Fig. 1. Equivalent mechanical model of the new device.

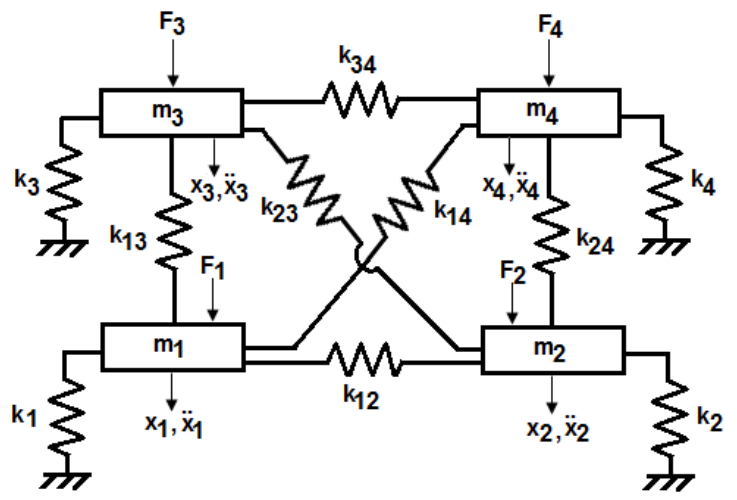

Fig. 2. Fabricated two-diaphragm Ormia-inspired microphone.

$f_{3}, f_{4}=\frac{1}{2 \pi} \sqrt{\frac{\left(C+K_{i}+2 K_{t 2}\right) M_{i}+\left(C+K_{o}+2 K_{t 1}\right) M_{o} \mp \sqrt{(A C+E+2 F)^{2}+4 B D^{2}}}{2 B}}$

where

$A=M_{i}-M_{o}, B=M_{i} M_{O}$,

$C=K_{c 1}+K_{c 2}, D=K_{c 1}-K_{c 2}$,

$E=K_{i} M_{i}-K_{o} M_{o}, F=K_{t 2} M_{i}-K_{t 1} M_{o}$,

and

$M_{o}=m_{1}=m_{2}, M_{i}=m_{3}=m_{4}$,

$K_{o}=k_{1}=k_{2}, K_{i}=k_{3}=k_{4}$,

$K_{t 1}=k_{t 12}, K_{t 2}=k_{t 34}$,

$K_{c 1}=k_{t 13}=k_{t 24}, K_{c 2}=k_{t 23}=k_{t 14}$.

After substituting the simulated values of the masses of the two diaphragms, the inherent stiffness and the coupling stiffness, the theoretical resonance frequencies for the first four mode shapes are $2104 \mathrm{~Hz}, 3619 \mathrm{~Hz}, 3826 \mathrm{~Hz}$ and 6447 
Hz. As $K_{c 1}, K_{c 2} \ll 0.5$, the coupling stiffness between the two diaphragms can be neglected, and the mechanical model is then simplified to two separated models which have their own rocking mode and bending mode, similar to Miles et al.'s original design. The rocking mode is the result of pressure difference between two wings, whereas the bending mode relates to the total pressure acting on the wings. Taking theouter square frame diaphragm for instance, with the same reflecting area as the inner circular diaphragm, the displacement of the frame is proportional to $P_{1}(t)-P_{2}(t)$ and $P_{1}(t)+P_{2}(t)$ at the rocking mode and the bending mode, respectively, where $P_{1}(t)$ is the pressure on the ipsilateral wing and $P_{2}(t)$ is pressure on the contralateral [7]. The time delay of an incident sound wave on to the two wings is $\tau=d \sin \theta / 2$, where $d$ is the distance between the centres of two wings and $\theta$ is azimuth angle of the incidence. Therefore,

$A_{\text {rocking }} \propto\left|e^{\frac{j \omega \tau}{2}}-e^{-\frac{j \omega \tau}{2}}\right| \propto\left|\sin \left(\frac{\omega d \sin \theta}{2 c}\right)\right|$

$A_{\text {bending }} \propto\left|e^{\frac{j \omega \tau}{2}}+e^{-\frac{j \omega \tau}{2}}\right| \propto\left|\cos \left(\frac{\omega d \sin \theta}{2 c}\right)\right|$.

Since the substrate is etched form the bottom, both the front and the rear surface of diaphragms are exposed to the air, working as a pressure gradient microphone. Assuming a diaphragm is mounted at two points and the pressure on the front surface equals $P_{0} e^{j w t-k x}$, where the $\mathrm{x}$-axis is the direction of propagation (i.e. the centre of diaphragm equates to $x=0)$ and $\mathrm{k}$ is the wave number, the pressure $F_{r}(t)$ on the rear surface can be expressed as [8]

$$
F_{r}(t)=P_{0} e^{j w t-k x}(1-j k L \cos \theta),
$$

where $\mathrm{L}$ is propagration path length of the sound wave. In addition, because the wave length is much longer than the width of the slits and the damping ratio in this model is small in order to reach higher sensitivity, the amplitude of displacement of wings at rocking mode and bending mode becomes

$A_{\text {rocking }} \propto\left|\sin \left(\frac{\omega d \sin \theta}{2 c}\right)\right| \propto \sin \theta$,

$A_{\text {bending }} \propto\left|\cos \left(\frac{\omega d \sin \theta}{2 c}\right)-1+j k L \cos \theta\right| \propto \cos \theta$,

when $\tau$ is very small (i.e. far field).

\section{SimUlation RESUlts}

Using COMSOL Multiphysics modelling software to simulate the working performance of the device model at $45^{\circ}$ sound incident angle, the amplitude spectrum below $10 \mathrm{kHz}$ was obtained as shown in Fig. 3. The first four simulated resonance frequencies are $2220 \mathrm{~Hz}, 3646 \mathrm{~Hz}, 3977 \mathrm{~Hz}$ and $6438 \mathrm{~Hz}$, which are strongly consistent with the previous mathematically derived values. Moreover, as expected, the spectrum reveals that the outer square frame mainly responds to sound at the first rocking mode frequency and the first bending mode frequency, while the inner circular plate can be used to detect sound direction near the second rocking mode and the second bending mode frequencies.

As can be seen from Fig. 4, this proves that the amplitude of displacement of the outer square frame has sine dependence at the first rocking mode and cosine behaviour at the first bending mode, as well as the amplitude of the circular diaphragm's displacement. However, a phase shift on the azimuth angle occurs at the second rocking mode when the circular plate localizes sound direction, which is primarily due to the coupling between two diaphragms.

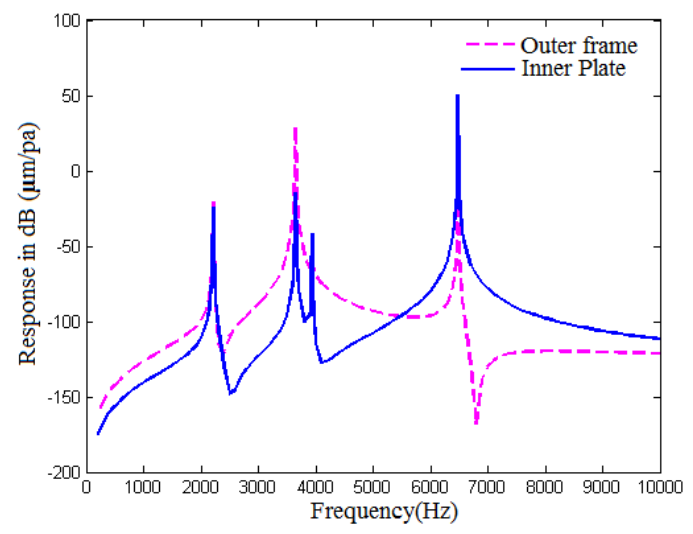

Fig. 3. Frequency response of outer frame and inner diaphragm at an incident angle of 45 degrees.

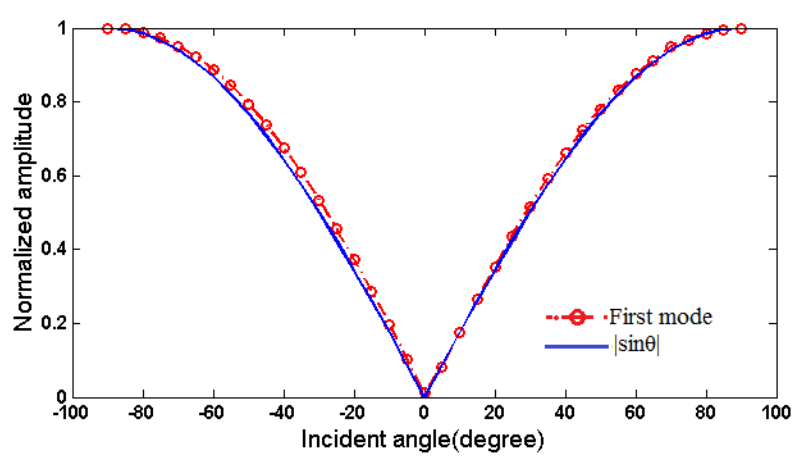

(a)

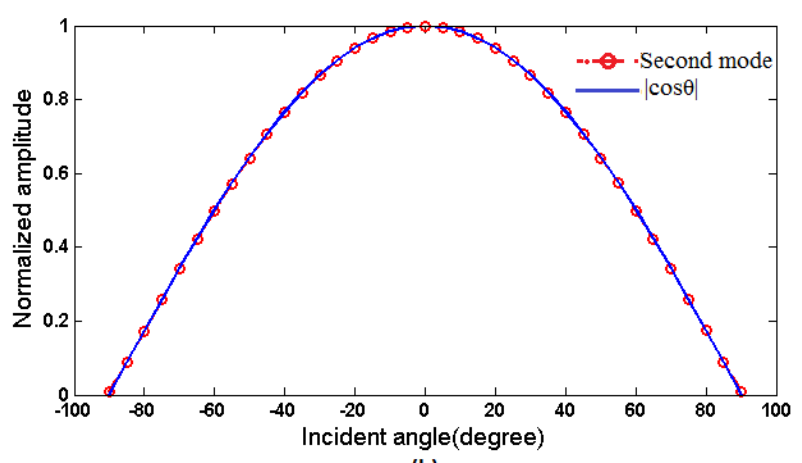

(b) 


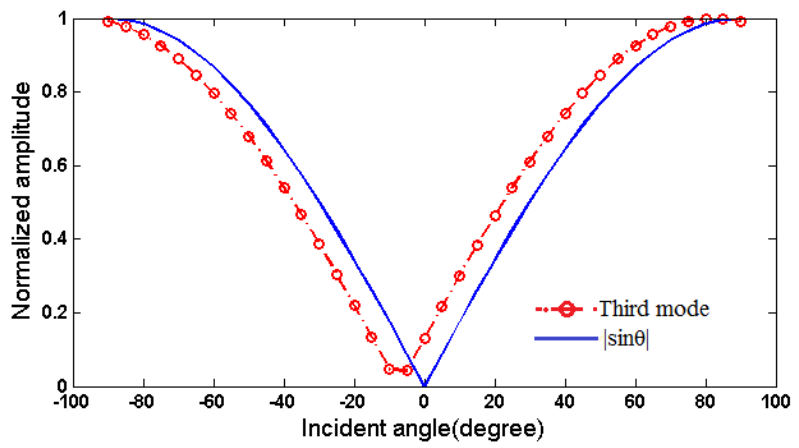

(c)

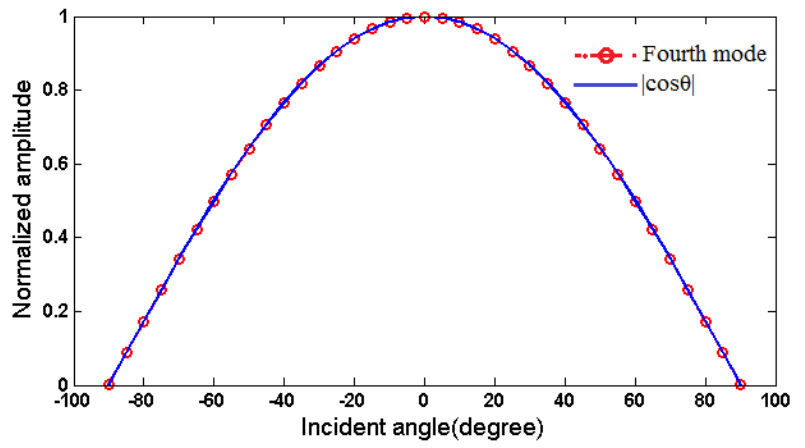

(d)

Fig. 4. Simulation results showing the relationship between normalized amplitudes at four resonance frequencies and trigonometric function: a) Amplitude of Point 1 at $2210 \mathrm{~Hz}$ (i.e. first mode), b) Amplitude of Point 1 at $3650 \mathrm{~Hz}$ (i.e. second mode), c) Amplitude of Point 3 at $3940 \mathrm{~Hz}$ (i.e. third mode), d) Amplitude of Point 3 at $6480 \mathrm{~Hz}$ (i.e. fourth mode).

\section{EXPERIMENT RESULTS}

The frequency response of the fabricated device was obtained using Laser Doppler vibrometer. The experimental values of the first four resonance frequencies are also indicated in TABLE I, and are compared with analysed values and the simulation values. The errors between the COMSOL results and practical values are mainly attributed to the tolerance in size and the position offset of the device. As the resonance frequencies of the second and the third mode are very close, these two frequency ranges may overlap when sound comes from some certain angles. Fig. 5 demonstrates first four observed modes shapes at 70 degrees azimuth angle.

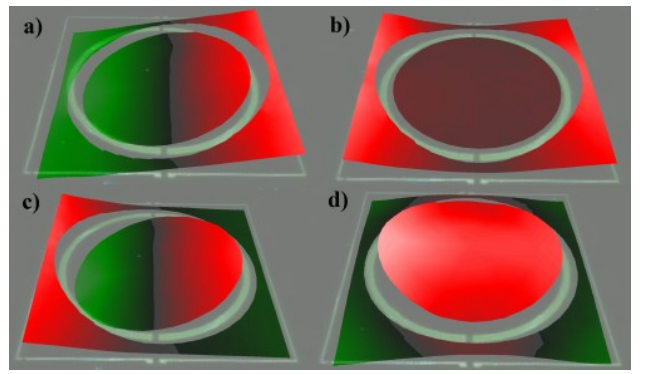

Fig. 5. Frequency response of outer frame and inner diaphragm at an incident angle of 70 degrees.
TABLE I. COMPARISON OF RESONANCE FREQUENCIES

\begin{tabular}{|l|c|c|c|c|}
\hline \multirow{2}{*}{ Methods } & \multicolumn{4}{|c|}{ Resonance Frequencies (Hz) } \\
\cline { 2 - 5 } & First & Second & Third & Fourth \\
\hline Calculation & 2104 & 3629 & 3826 & 6447 \\
\hline Simulation & 2220 & 3646 & 3977 & 6438 \\
\hline Experiment & 1975 & 3594 & 3738 & 7031 \\
\hline
\end{tabular}

\section{CONCLUSION}

The earlier Ormia-inspired MEMS directional microphone designs can detect sound direction in no more than two frequency bands. The design model presented in this paper increases the number of working frequency bands, by using two concentric diaphragms. These respond directionally with sine or cosine functions as verified by COMSOL simulation. The new design has smaller dimensions and less fabrication complexity than a microphone array based on earlier designs. This is part due to the use of SOIMUMPS. The new design would also require less post-processing to reduce errors when localizing sound sources across multiple frequency bands. In the future, the structure will be optimized to approach the distinct second resonance frequency and the third resonance frequency. Moreover, a backplate will be attached to the bottom of the etched substrate in order to increase air damping and enhance the directional ability based on measuring phase difference of displacements of each diaphragm.

\section{ACKNOWLEDGMENTS}

This research is supported by University of Strathclyde. Yansheng Zhang thanks James Brown in Centre for Microsystems and Photonics and Andrew Reid in Centre for Ultrasonic Engineering for taking an image of the fabricated device.

\section{REFERENCES}

[1] L. E. Kinsler, A. R. Frey, A. B. Coppens, J. V. Sanders, Fundamentals of Acoustics, 4th ed., New York: John Wiley \& Sons, 2000, pp. 416-417

[2] D. Robert, R. N. Miles, and R. R. Hoy, "Tympanal mechanics in the parasitoid fly Ormia ochracea: intertympanal coupling during mechanical vibration," Journal of Comparative Physiology A-Sensory Neural and Behavioral Physiology, vol. 183, no. 4, pp. 443-452, October, 1998.

[3] B. Bicen, S. Jolly, K. Jeelani et al., "Integrated optical displacement detection and electrostatic actuation for directional optical microphones with micromachined biomimetic diaphragms," IEEE Sensors Journal, vol. 9, no. 12, pp. 1933-41, December, 2009.

[4] M. Touse, J. Sinibaldi, K. Simsek et al., "Fabrication of a microelectromechanical directional sound sensor with electronic readout using comb fingers," Applied Physics Letters, vol. 96, no. 17, pp. 1-3, Apr 26, 2010.

[5] H. Liu, L. Currano, D. Gee et al., "Understanding and mimicking the dual optimality of the fly ear," Scientific Reports, vol. 3, Augest 22, 2013.

[6] "MEMSCAP | PolyMUMPs and MEMS multi project wafer Service," http://www.memscap.com/products/mumps/polymumps.

[7] R. N. Miles, D. Robert, and R. R. Hoy, "Mechanically coupled ears for directional hearing in the parasitoid fly Ormia ochracea," Journal of the Acoustical Society of America, vol. 98, no. 6, pp. 3059-3070, Dec, 1995.

[8] D. Davis, and J. Eugene Patronis, Sound system engineering, 3rd ed., New York: Elsevier Focal Press, 2006, pp. 233-239, 
